\title{
An Open Platform for 3D Face Recognition Algorithms
}

\author{
Hassen DRIRA ${ }^{\mathrm{a}}$, Boulbaba BEN AMOR ${ }^{\mathrm{b}, \mathrm{a}}$, Mohamed DAOUDI ${ }^{\mathrm{b}, \mathrm{a}}$, Anuj SRIVASTAVA ${ }^{\mathrm{c}}$, \\ and Joseph COLINEAU ${ }^{d}$ \\ aLIFL (UMR CNRS 8022), Université de Lille1, France ; \\ ${ }^{b}$ Institut TELECOM, TELECOM Lille 1, France ; \\ 'Departement of Statistics, FSU, Tallahassee, FL 32306, USA ; \\ ${ }^{\mathrm{d}}$ THALES-TRT, 91767 Palaiseau, France.
}

\begin{abstract}
In this paper we describe a new open platform designed to integrate 3D face matching algorithms for recognition. Its main purpose is to provide experimental environment to online operational testing of 3D face recognition approaches, in laboratory conditions. The proposed platform consists of: (i) An acquisition module that interfaces with Minolta 3D laser-based scanner, (ii) A preprocessing sub-system allowing detection and segmentation of the useful part of the face from the depth image (scanner's output) and its processing (iii) A face matching module that incorporates matching algorithms, and (iv) A decision component that provides the final matching result. Moreover, we show an integration example of our algorithm [1] and discuss experimental results. Our 3D facial matching algorithm currently integrated to the proposed platform represents facial surfaces by indexed collections of radial curves on them, emanating from the nose tips, and compares the facial shapes by comparing the shapes of their corresponding curves. Using a framework on elastic shape analysis of curves, we obtain an algorithm for comparing facial surfaces. We also introduce a quality control module which allows our approach to be robust to pose variations and missing data. Comparative evaluation using a common experimental setup on GavabDB ${ }^{1}$ dataset, considered as the most expression-rich and noise-prone 3D face dataset, shows that our approach outperforms other state-of-the-art approaches.
\end{abstract}

Keywords: 3D face matching, face recognition, open platform, preprocessing, geodesics.

\section{Introduction}

The various tools that are called Biometric Technologies are simply means physiological characteristics, human body parts and their appearances, used to recognize individual human beings in the course of daily activities. The appearances of body parts, especially in imaged data, have a large variability and are influenced by their shapes, colors, illumination environment, presence of other parts, and so on. Therefore, the researchers have focused on body parts and images that minimize this variability within class (persons) and maximize it across classes. Since 2D (visible light) images of faces are greatly susceptible to variations in the imaging environments (camera pose, illumination patterns, etc.), the researchers have argued for the need to use 3D face data, typically collected by laser scanners, for studying shapes of peoples' faces and using this shape analysis for biometrics. The outputs from laser scanners are minimally dependent on the external environmental factors and provide faithful measurements of shapes of facial surfaces. The only remaining variability that is manifested within the same class, i.e. within the measurements of the same person, is the one introduced by changes in facial expressions and significant pose variations, in addition to the data quality. Fig. 1 illustrates main 3D face recognition challenges.

Facial expressions, such as smile, serious, fear, and anger, are prime indicators of the emotional state of a person and, thus, are important in estimating mood of a person, for example in developing intelligent ambient systems, but may have a lesser role in biometric applications. In fact, variations in facial expressions change the shapes of facial surfaces to some extent and introduce a detrimental variability that has to be accounted for in shape-based 3D face recognition. The other important issue is related to data collection and imperfections introduced in that process. It is difficult to obtain a pristine, continuous facial surface, or a mesh representing such a surface, with the current laser technology. One typically gets holes in the scanned data in locations of eyes, lips, and outside regions. For instance, scans of people with open mouths result in holes in the mouth region.

\footnotetext{
${ }^{1}$ http://www.gavab.es/recursos_en.html
} 


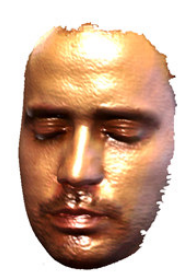

Neutral frontal scan

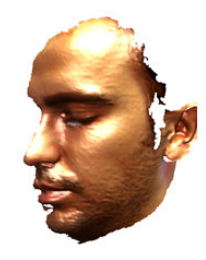

(a) pose variations

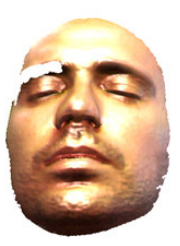

Fig. 1. the main 3D face recognition challenges.

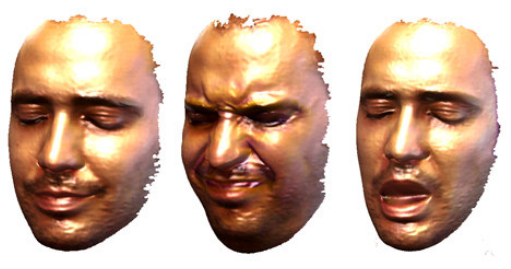

(b) expression variations

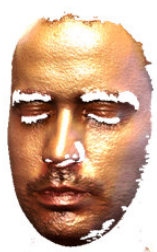

(c) data quality

Existing 3D face recognition methods can be classified into two categories. First, some methods, such as Mousavi et al. [12], Mahoor et al. [10], and Li et al. [9], are interested in adopting low-level geometric features to face recognition for several reasons. They claim that they spend less effort in extracting features unlike the methods that are based on extracting high-level features, e.g., shapes of facial curves [14], concave and convex facial regions [2], partial face regions [6][11], or deformation distance metrics [8]. The latest methods constitute the second category, and their recognition performance generally depends on the reliability of the features. For this reason, it is necessary to spend effort to extract these features and look for elaborate tools to compare them. For example, shape analysis can be a good candidate especially it has shown good performance in previous works [14]. In Samir et al. [15], the level curves of the surface distance function that resulted in 3D curves are used. The authors used a non-elastic metric and a path-straightening method to compute geodesics between these curves. Here the matching was not studied and the correspondence of curves and points across faces was simply linear. However, the open mouth corrupts the shape of some level curves and this parameterization does not handle this problem. To avoid this problem of open mouth, Drira et al. [4] proposed partial biometrics. That is, they studied the contribution of only the nasal region in 3D face recognition using a similar level curves-based approach. They used elastic matching for curves comparison. Mpiperis et al. [13] proposed a geodesic polar parameterization of the face surface. With this parameterization, the intrinsic surface attributes do not change under isometric deformations when the mouth is closed. When the mouth is open it violates the isometry assumption and they modify their geodesic polar parameterization by disconnecting the lips. Therefore, their approach requires lips detection. Bronstein et al. [4] use multi-dimensional scaling on pair-wise geodesic distance to embed the surface to a 4D shpere were classification is performed on the basis of normalized moments. As [13], their approach requires also lips detection. Berretti et al. in [2] and [3], proposed to use features derived from the surface paths around the nose. In [3], the radial curves were extracted as features, and then matched using SVM classifiers. In [2], a compact graph representation is constructed for each face. In this way, the structural similarity between two face models is evaluated by matching their corresponding graphs. In our approach described in more details in [1], we explore the use of shapes of elastic radial curves to model 3D facial deformations, caused by changes in facial expressions. First, we extract radial curves as facial features. Then, we apply elastic shape analysis in order to keep the intrinsic surface attributes under isometric deformations even when the mouth is open. Unlike previous works dealing with large facial expressions, especially when the mouth is open [13][4] which require lips detection, our approach mitigates this problem without any lip detection.

The main contribution of this paper is to present a new open platform designed to incorporate 3D facial matching algorithms and allows their operational testing. The rest of the paper is organized as follows. Next, section 2 gives an overview of the proposed platform with an integration example of our facial surfaces comparison algorithm. In section 3, we describe the common data pre-processing module. Section 4 describes the principles of our Riemannian framework to analyze shapes of radial curves and its extension to compare facial surfaces. We demonstrate in section 5 the performance to recognize people in presence of both expression and pose variations. The section 6 provides some concluding remarks.

\section{Overall platform architecture}

This section describes all modules of the platform and proposes an integration example of our algorithm in that platform. Fig.2 shows the environment of the developed platform (software) including the Minolta 3D laser-based scanner and the RFID-based reader system. The developed software interfaces with the 3D scanner and the RFID system using their Software Development Kits. All 3D mesh processing steps are developed following an oriented object programming process using the $\mathrm{C}++$ language. 


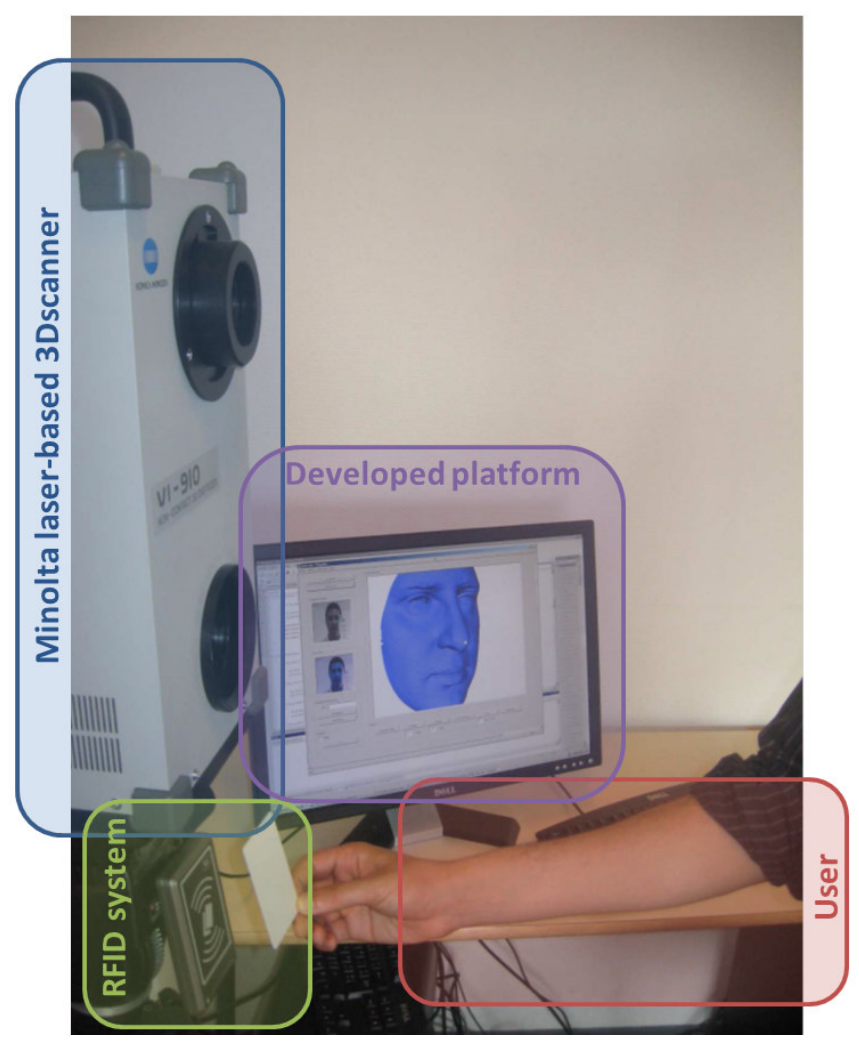

Fig. 2. The developed platform (software) in his testing environment (laboratory conditions).

After their acquisition by interfacing with the Minolta scanner, the probe image $\mathbf{P}$ and the gallery image $\mathbf{G}$ acquired images are pre-processed. This step is essential to improve the quality of the depth images and to extract the useful part of the face. It consists of a Laplacian smoothing filter to reduce the acquisition noise, a filling hole filter that identifies and fills holes in input mesh, and a cropping filter that cuts and returns the part of the input mesh inside of a specified sphere. The preprocessing module outputs 3D meshes of the useful part of the faces to be compared that present the inputs of the matching algorithm.

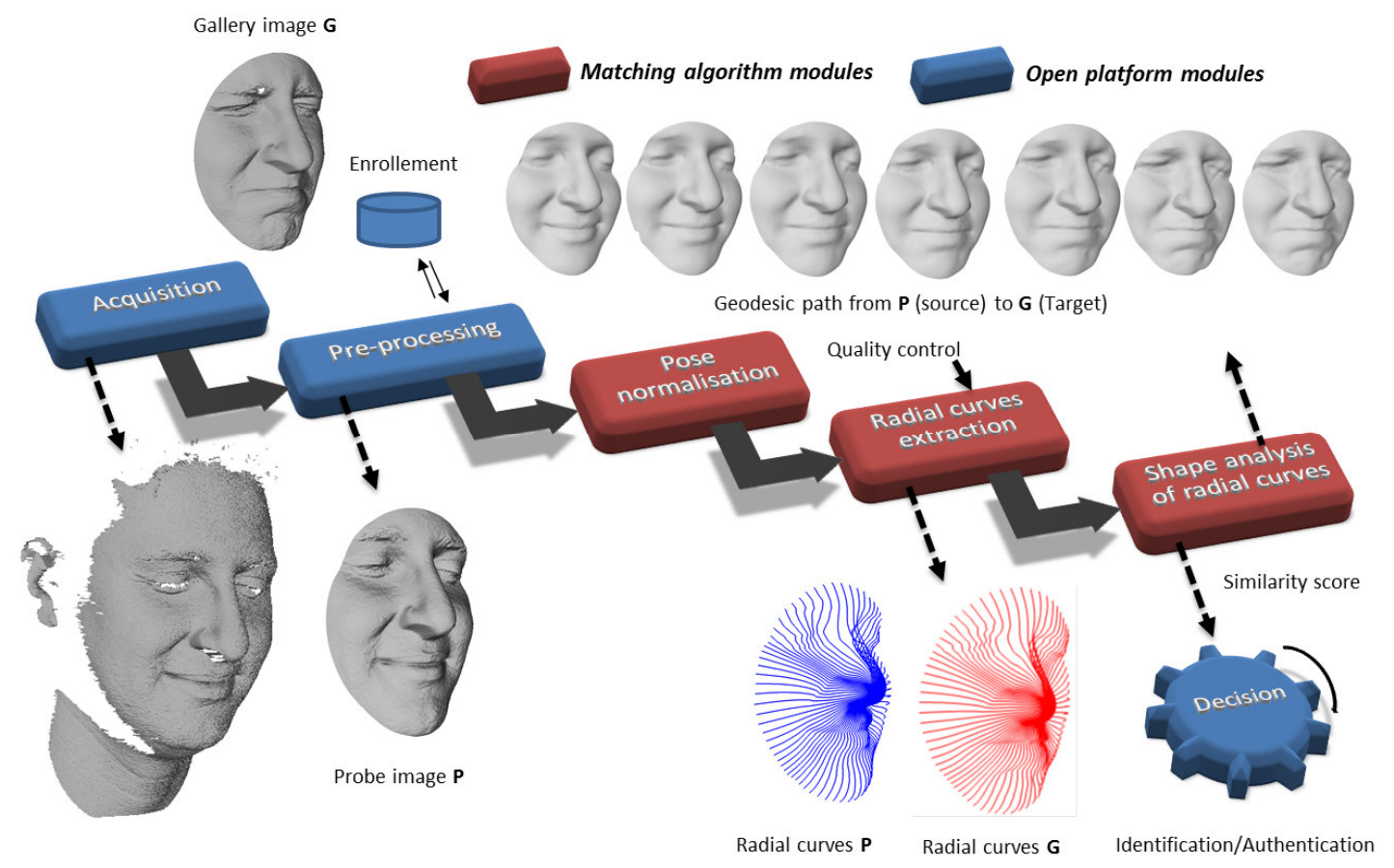

Fig. 3. Overview of the proposed platform with our matching algorithm [1] integration. 
In order to compare probe and gallery faces, our matching algorithms correct their poses by performing coarse then fine alignments. The coarse alignment is performed based on the translation vector formed by the tips of the noses. This step is followed by a finer alignment based on the well-known ICP algorithm in order to correct their pose. Next, we extract the radial curves emanating from the nose tip and having different directions on the face. Within this step, a quality control module inspects the quality of each curve on both meshes and keeps only the good ones based on defined criteria [1]. In order to improve matching and comparisons between the extracted curves, we advocate the use of elastic matching. Actually, facial deformations due to expressions can be attenuated by an elastic matching between facial curves. Hence, we obtain algorithm for computing geodesics between pairwise of radial curves on gallery and probe meshes. The length of one geodesic measures the degree of similarity between one pair of curves. The fusion of the scores on good quality common curves, produced similarity score between the faces $\mathbf{P}$ and $\mathbf{G}$. Fig. 3 illustrates the pipeline of modules that form the proposed platform with an integration example of our matching algorithm [1].

\section{3D data pre-processing}

This module belongs to the open platform as essential step to improve the quality of input depth images and to discard undesired parts (clothes, neck, ears, hair, etc.). Indeed, these images outputs of laser scanners present some imperfections as holes, spikes and so on. As illustrated in Fig. 4, this step consists of a pipeline of 3D mesh processing filters:

i. Filling holes filter identifies and fills holes in input meshes. Holes are created either because of the absorption of laser in dark areas such as eyebrows and mustaches, occlusion or mouth opening. They are identified in the input mesh by locating boundary edges, linking them together into loops, and then triangulating the resulting loops.

ii. Cropping filter cuts and returns parts of the mesh inside a defined implicit function. This function is a sphere defined by the nose tip as its center and the radius $75 \mathrm{~mm}$ in order to avoid as much hair.

iii. Smoothing filter reduces high frequency information (spikes) in the geometry of the mesh, making the cells better shaped and the vertices more evenly distributed.

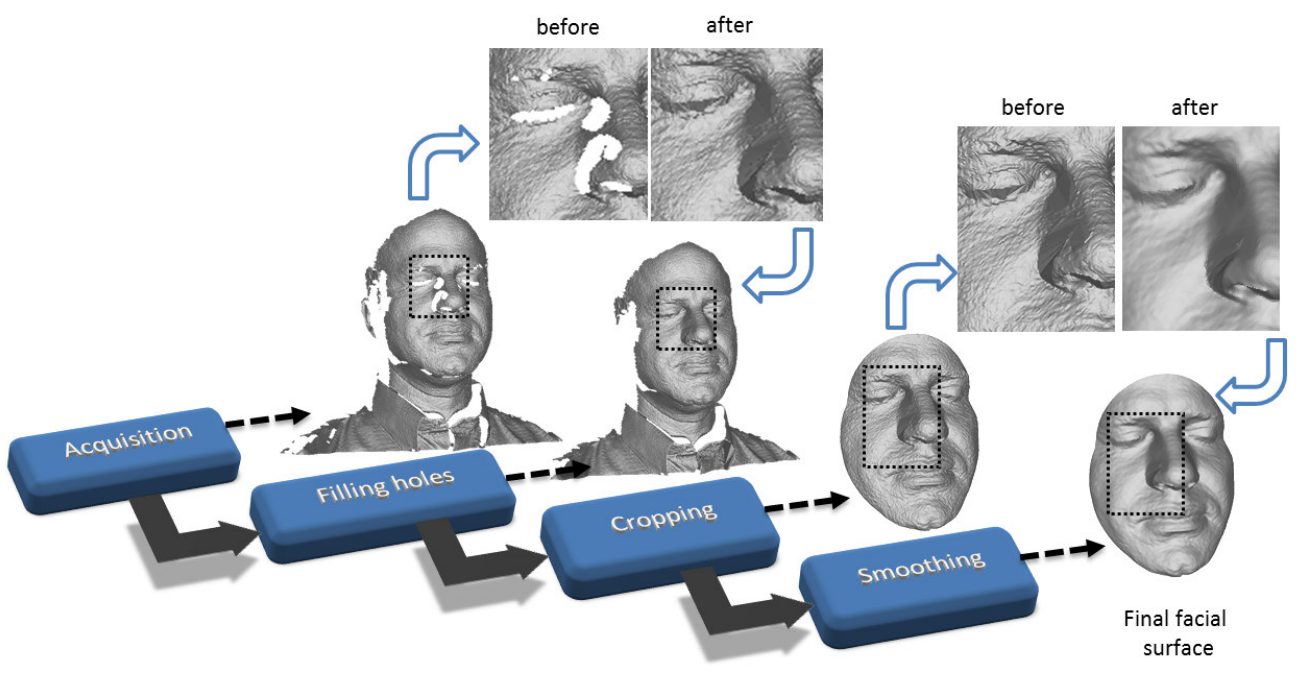

Fig. 4. Pipeline of filters in the pre-processing steps.

The development of the pre-processing module is based on The Visualization ToolKit ${ }^{2}$ routines. VTK consists of a $\mathrm{C}++$ class library and provides a suite of mesh processing filters.

\section{Facial shapes analysis and comparison module}

After data pre-processing, our goal is to compare shapes of facial surfaces using their facial radial curves. In other words, we represent surfaces by an indexed collection of simple, open curves in $\mathbb{R}^{3}$ and the geometry of a surface is then studied using the geometries of the associated curves [1]. Let $S$

\footnotetext{
${ }^{2}$ http://www.vtk.org/
} 
be a facial surface denoting the output of the previous preprocessing step. Although $S$ is a triangulated mesh, we start the discussion by assuming that it is a continuous surface. Let $\beta_{\alpha}$ denote the radial curve which make an angle $\alpha$ with a reference radial curve. The reference curve is chosen to be the vertical curve once the face has been rotated to the upright position. In practice, each radial curve $\beta_{\alpha}$ is obtained by slicing the facial surface by a plane $P_{\alpha}$ that has the nose tip as origin and makes an angle $\alpha$ with the plane containing the reference curve. That is, the intersection of $P_{\alpha}$ with $S$ gives $\beta_{\alpha}$. We repeat this step to extract all radial curves from the facial surface at equal angular separation. This indexed collection of radial curves, captures the shape of a facial surface and forms our mathematical representation of that surface. We have chosen to represent a surface with a collection of curves since we have better tools for analyzing shapes of curves than we have for surfaces. More specifically, we are going to use an elastic framework for studying shapes of curves that is especially suited to modeling deformations associated with changes in facial expressions. The shape analysis-based comparison of the radial curves is detailed in [1].
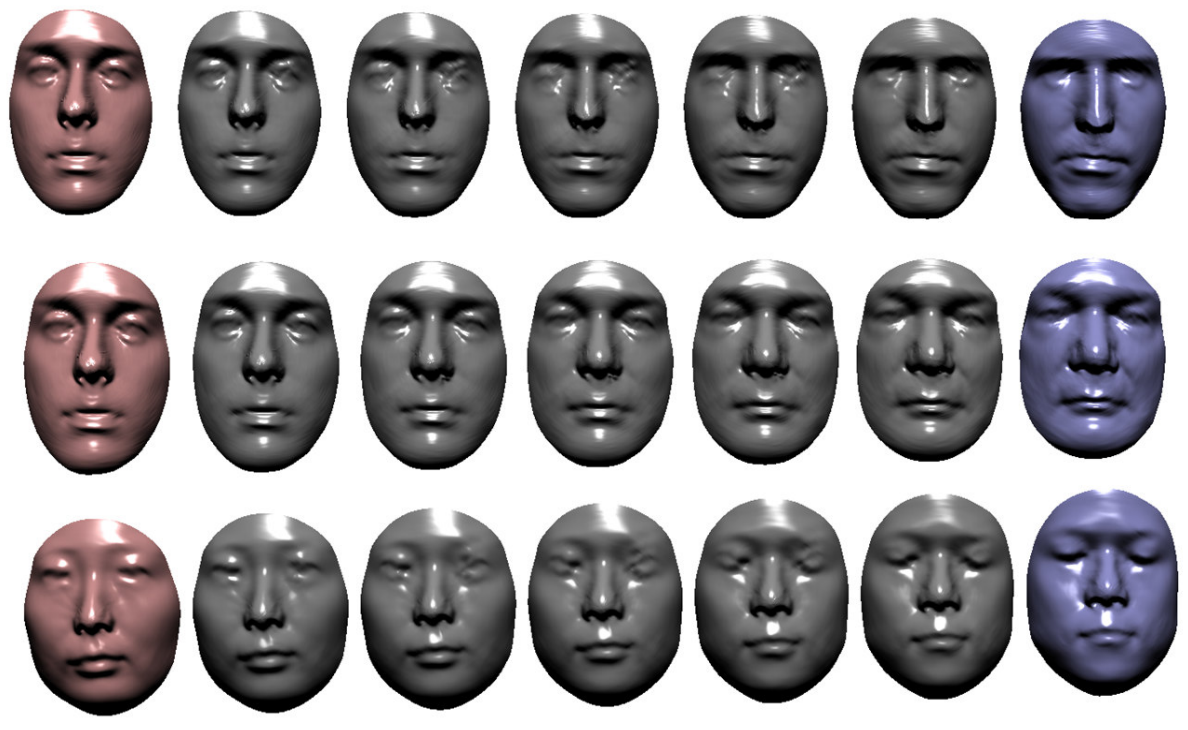

Fig. 5. Examples of inter-class geodesics (different subjects).
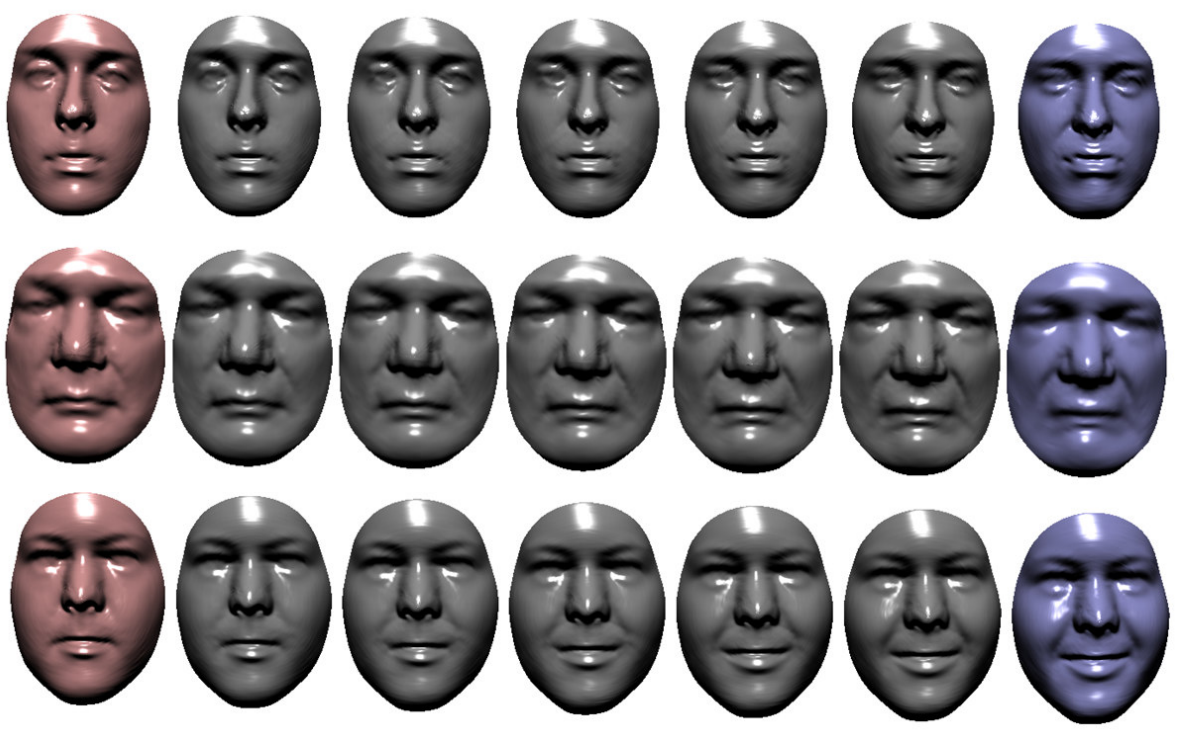

Fig. 6. Examples of intra-class geodesics (same subject, different expressions).

Since we have geodesic paths denoting optimal deformations between individual curves [1], we can combine these deformations to obtain full deformations between faces. In fact, these full deformations are geodesic paths between faces when represented. As mentioned earlier, we are going to represent a face surface $S$ with an indexed collection of radial curves. The indexing provides a correspondence between curves across faces. For example, a curve at an angle $\alpha$ on face 1 is matched with the curve at the same angle on face 2. Shown in Fig.5 and Fig.6 are examples of such geodesic paths between source and target faces. It is clear that the geodesic in the shape space models in a naturel way the 
deformation from the source to the target, especially in mouth region. We present the illustrations in both cases; case of inter-class path (source and target belong to different persons) and intra-class path (source and target belong to the same person). 3D faces used in this illustration are taken from FRGCv2 dataset [16]. This computation provides several quantities of interest. Firstly, if we consider each facial curve as an independent feature, then we get a metric for each feature. We can then use any metric-based classifier of these features to result in a feature-by-feature classification of faces. On other words, we can use individual radial curves on faces for classifying people. Secondly, it provides a Riemannian distance between shapes of full facial surfaces by combining distances between the corresponding radial curves. Thirdly, since we have geodesic paths denoting optimal deformations between individual curves, we can combine these deformations to obtain full deformations between faces. In this work, we define the distance between faces as a combination of individual distances [1].

\section{Experiments and evaluations}

Our algorithm for representation and matching of 3D faces has been evaluated using the GavabDB database. To the best of our knowledge, GavabDB is the most expression-rich and noise-prone 3D face dataset currently available to the public. The GavabDB consists of Minolta Vi-700 laser range scans from 61 different subjects. The subjects, of which 45 are male and 16 are female, are all Caucasian. Each subject was scanned 9 times for different poses and expressions, namely six neutral expression scans and three scans with an expression. The neutral scans include two different frontal scans, one scan while looking up (+35 degree), one scan while looking down (-35 degree), one scan from the right side (+90 degree), and one from the left side (-90 degree). The expression scans include one with a smile, one with a pronounced laugh, and an arbitrary expression freely chosen by the subject (see Fig.7).
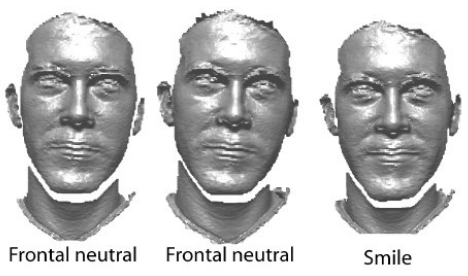

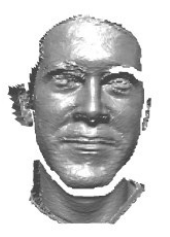

Accentuated laugh Random gesture

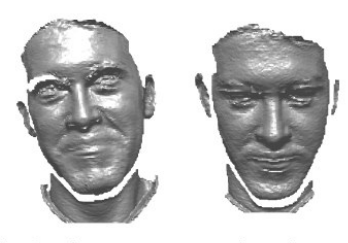

Looking down

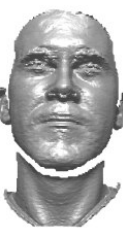

Looking up
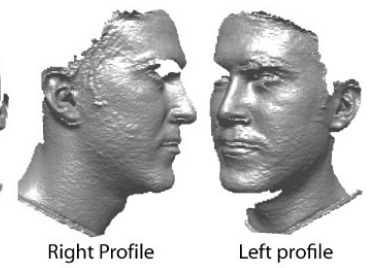

Fig. 7. Examples of 3D scans of the same subject from Gavab dataset.

In our experiments, one of the two frontal models with the neutral expression provided for each person is taken as a gallery model whereas the rest are considered as probe images.

Table.1. Comparison of recognition rates using different methods : (a) Neutral, (b) Expressive, (c) Neutral+expressive, (d) Rotated looking down, (e) Rotated looking up, (f) Overall, (g) Scans from right sight, (g) Scans from left sight.

\begin{tabular}{|l|l|l|l|l|l|l|}
\hline & Li et al. [8] & Moreno et al. [10] & Mahoor et al. [9] & Berretti et al.[1] & Mousavi et al. [11] & Our \\
\hline (a) & $96.67 \%$ & $90.16 \%$ & $95 \%$ & $94 \%$ & - & $100 \%$ \\
\hline (b) & $93.33 \%$ & $77.9 \%$ & $72 \%$ & $81 \%$ & - & $94.54 \%$ \\
\hline (c) & $94.68 \%$ & - & $78 \%$ & $84.25 \%$ & $91 \%$ & $94.67 \%$ \\
\hline (d) & - & - & $85.3 \%$ & $80 \%$ & - & $100 \%$ \\
\hline (e) & - & - & $88.6 \%$ & $79 \%$ & - & $98.36 \%$ \\
\hline (f) & - & - & - & $82.66 \%$ & $81.67 \%$ & $96.99 \%$ \\
\hline (g) & - & - & - & - & - & $70.49 \%$ \\
\hline (h) & - & - & - & - & - & $86.89 \%$ \\
\hline
\end{tabular}

Table 1 illustrates the results of the matching accuracy for different categories of probe faces. We notice that our approach provides high recognition accuracy for expressive faces (94.54\%) comparing to state-of-the-art approaches. This is due to both face parameterization using radial curves and elastic matching techniques. Actually, each curve represents a feature that characterizes a local region in the face contrary to the parameterization using closed level curves. Besides, the elastic matching is able to establish a correspondence with guaranteed alignment among anatomical facial features. As far as neutral expressions are concerned, the accuracy depends on the pose. Actually, the accuracy is not affected when the pose is frontal or rotated looking down (the recognition rate is $100 \%$ ). According to scans from right and left sides, the accuracy is not very high as missing data are significant. To the best of our knowledge, no earlier work included scans from left and right sights in their experimental protocol when using Gavab dataset. Therefore, we show in table 1 comparison with other methods on a subset of Gavab dataset (without these scans). Notice that the experimental protocol is the same; 
one of the two frontal neutral scan is chosen as gallery, remaining faces constitute the probe dataset. As shown in the table 1, our method outperforms all other ones in terms of recognition rates.

Table.2. Computational cost of the proposed approach to compare two $3 D$ faces

\begin{tabular}{|l|l|}
\hline Step & Time consumed (s) \\
\hline Mesh preprocessing & 0.375 (per face) \\
\hline Mesh alignment & 0.125 \\
\hline Curves extraction & 0.485 (all curves, 2 faces) \\
\hline Quality control & 0.000001875 (per curve) \\
\hline Curve preprocessing & 0.137 (per curve) \\
\hline Curve comparison & 0.0318 (per curve) \\
\hline Overall & 7.7 \\
\hline
\end{tabular}

Table 2 presents the time consumed for different steps of the algorithm on a PC with a 3 Ghz Core 2 Duo processor with 3 GB memory. In order to assess the effectiveness of the proposed solution for face identification, we performed extensive experiments. In these experiments, one of the two frontal models with the neutral expression provided for each person is taken as a gallery model for the identification.

\section{Conclusions}

In this paper we developed an open platform for 3D face recognition algorithms. This platform provides an acquisition module which interfaces with the Minolta 3D scanner and a pre-processing sub-system that extracts and prepares the useful part of the face for the matching step. Moreover, we describe an original solution for 3D face matching under facial expressions and poses changes, integrated to the proposed platform. This platform contributes to research development in 3D face biometric field by providing tools for operational testing of developed algorithms.

\section{Acknowledgements}

This research is supported in part by the ANR under the project ANR-07-SESU-004 and the Contrat de Projet Etat-Région (CPER) Région Nord-Pas de Calais Ambient Intelligence and partially supported by the following grants: ARO W911NF-04-01-0268 and AFOSR FA9550-06-1-0324 to Anuj Srivastava. Additionally, Anuj Srivastava was supported by visiting professorships from University of Lille I and CNRS in summers of 2007-2009.

\section{References}

1. Hassen Drira, Boulbaba Ben Amor, Mohamed Daoudi, and Anuj Srivastava. Pose and Expression-Invariant 3D Face Recognition using Elastic Radial Curves. In Proceedings of the British Machine Vision Conference, pages 90.1-90.11, 2010.

2. Stefano Berretti, Alberto Del Bimbo, and Pietro Pala. 3d face recognition by modelling the arrangement of concave and convex regions. In Proceedings of Adaptive Multimedia Retrieval, pages 108-118, 2006.

3. Stefano Berretti, Alberto Del Bimbo, Pietro Pala Francisco Silva-Mata. Face recognition by svms classification of $2 \mathrm{D}$ and $3 \mathrm{D}$ radial geodesics. In Proceedings of International Conference on Multimedia \& Expo, pages 93-96, 2008.

4. Alexander M. Bronstein, Michael M. Bronstein, and Ron Kimmel. Expression invariant representations of faces. IEEE Transactions on Image Processing, 16(1): 188-197, 2007.

5. Hassen Drira, Boulbaba Ben Amor, Anuj Srivastava, and Mohamed Daoudi. A Riemannian analysis of $3 \mathrm{~d}$ nose shapes for partial human biometrics. In Proceedings of International Conference on Computer Vision, pages 2050-2057, 2009.

6. Timothy C. Faltemier, Kevin W. Bowyer, and Patrick J. Flynn. A region ensemble for 3-d face recognition. IEEE Transactions on Information Forensics and Security, 3(1): 62-73, 2008.

7. Shantanu H. Joshi, Eric Klassen, Anuj Srivastava, and lan Jermyn. A novel representation for Riemannian analysis of elastic curves in $I R^{n}$. In Proceedings of International Conference on Computer Vision and Pattern Recognition, 2007.

8. I. A. Kakadiaris, G. Passalis, G. Toderici, M. N. Murtuza, Y. Lu, N. Karampatziakis, and T. Theoharis. Three-dimensional face recognition in the presence of facial expressions: An annotated deformable model approach. IEEE Transactions on Pattern Analysis and Machine Intelligence, 29(4):640-649, 2007. 
9. Xiaoxing $\mathrm{Li}$, Tao Jia, and Hao Zhang. Expression-insensitive $3 \mathrm{~d}$ face recognition using sparse representation. Computer Vision and Pattern Recognition, In Proceedings of International Conference on Computer Vision and Pattern Recognition, 0:2575-2582, 2009.

10. Mohammad $\mathrm{H}$. Mahoor and Mohamed Abdel-Mottaleb. Face recognition based on $3 \mathrm{~d}$ ridge images obtained from range data. Pattern Recognition, 42(3):445-451, 2009.

11. Ana Belen Moreno, Angel Sanchez, Jose Fco. Velez, and Fco. Javier Díaz. Face recognition using 3d local geometrical features: Pca vs. svm. In Int. Symp. on Image and Signal Processing and Analysis, 2005.

12. Mir Hashem Mousavi, Karim Faez, and Amin Asghari. Three dimensional face recognition using svm classifier. In Proceedings of the Seventh IEEE/ACIS International Conference on Computer and Information Science, pages 208-213, Washington, DC, USA, 2008.

13. Iordanis Mpiperis, Sotiris Malassiotis, and Michael G. Strintzis. 3-d face recognition with the geodesic polar representation. IEEE Transactions on Information Forensics and Security, (3-2):537-547.

14. Chafik Samir, Anuj Srivastava, and Mohamed Daoudi. Three-dimensional face recognition using shapes of facial curves. IEEE Transactions on Pattern Analysis and Machine Intelligence, 28:1858-1863, 2006. ISSN 0162-8828.

15. Chafik Samir, Anuj Srivastava, Mohamed Daoudi, and Eric Klassen. An intrinsic framework for analysis of facial surfaces. International Journal of Computer Vision, 82(1):80-95, 2009.

16. P. Jonathon Phillips, Patrick J. Flynn, Todd Scruggs, Kevin W. Bowyer, Jin Chang, Kevin Hoffman, Joe Marques, Jaesik Min, and William Worek. Overview of the face recognition grand challenge. In Proceedings of the IEEE Computer Society Conference on Computer Vision and Pattern Recognition (CVPR'05) - Volume 1, pages 947-954, 2005. 